\title{
Citizen science reveals widespread negative effects of roads on amphibian distributions
}

\author{
Bradley J. Cosentino ${ }^{\mathrm{a}, *}$, David M. Marsh ${ }^{\mathrm{b}}$, Kara S. Jones ${ }^{\mathrm{c}}$, Joseph J. Apodaca ${ }^{\mathrm{d}}$, Christopher Bates ${ }^{\mathrm{e}}$, \\ Jessica Beach $^{\mathrm{f}}$, Karen H. Beard ${ }^{\mathrm{g}}$, Kelsie Becklin ${ }^{\mathrm{h}}$, Jane Margaret Bell ${ }^{\mathrm{d}}$, Christopher Crockett ${ }^{\mathrm{i}}$, \\ George Fawson $^{\mathrm{g}}$, Jennifer Fjelsted ${ }^{\mathrm{i}}$, Elizabeth A. Forys ${ }^{\mathrm{j}}$, Kristen S. Genet ${ }^{\mathrm{h}}$, Melanie Grover ${ }^{\mathrm{d}}$, \\ Jaimie Holmes ${ }^{k}$, Katherine Indeck ${ }^{j}$, Nancy E. Karraker ${ }^{k}$, Eran S. Kilpatrick ${ }^{\mathrm{e}}$, Tom A. Langen ${ }^{\mathrm{f}}$, \\ Stephen G. Mugel ${ }^{\mathrm{a}}$, Alessandro Molina ${ }^{\mathrm{i}}$, James R. Vonesh ${ }^{\mathrm{i}}$, Ryan J. Weaver ${ }^{\mathrm{i}}$, Anisha Willey ${ }^{\mathrm{g}}$
}

a Department of Biology, Hobart and William Smith Colleges, Geneva, NY 14456, USA

${ }^{\mathrm{b}}$ Department of Biology, Washington and Lee University, Lexington, VA 24450, USA

${ }^{\mathrm{c}}$ Department of Environmental Science and Policy, George Mason University, Fairfax, VA 22030, USA

${ }^{\mathrm{d}}$ Department of Environmental Studies and Biology, Warren Wilson College, Asheville, NC 28815, USA

${ }^{\mathrm{e}}$ Division of Mathematics and Science, University of South Carolina Salkehatchie, Walterboro, SC 29488, USA

${ }^{\mathrm{f}}$ Department of Biology, Clarkson University, Potsdam, NY 13699, USA

${ }^{\mathrm{g}}$ Department of Wildland Resources and the Ecology Center, Utah State University, Logan, UT 84322, USA

${ }^{\mathrm{h}}$ Department of Biology, Anoka Ramsey Community College, Coon Rapids, MN 55433, USA

${ }^{i}$ Department of Biology, Virginia Commonwealth University, Richmond, VA 23284, USA

${ }^{\mathrm{j}}$ Department of Environmental Science and Biology, Eckerd College, St. Petersburg, FL 33711, USA

${ }^{\mathrm{k}}$ Department of Natural Resources Science, University of Rhode Island, Kingston, RI 02881, USA

\section{A R T I C L E I N F O}

\section{Article history:}

Received 3 June 2014

Received in revised form 1 September 2014

Accepted 20 September 2014

Available online 11 October 2014

\section{Keywords:}

Amphibian

Habitat loss

Habitat fragmentation

Landscape structure

Road traffic

Urbanization

\begin{abstract}
A B S T R A C T
Landscape structure is important for shaping the abundance and distribution of amphibians, but prior studies of landscape effects have been species or ecosystem-specific. Using a large-scale, citizen science-generated database, we examined the effects of habitat composition, road disturbance, and habitat split (i.e. the isolation of wetland from forest by intervening land use) on the distribution and richness of frogs and toads in the eastern and central United States. Undergraduates from nine biology and environmental science courses collated occupancy data and characterized landscape structure at 1617 sampling locations from the North American Amphibian Monitoring Program. Our analysis revealed that anuran species richness and individual species distributions were consistently constrained by both road density and traffic volume. In contrast, developed land around wetlands had small, or even positive effects on anuran species richness and distributions after controlling for road effects. Effects of upland habitat composition varied among species, and habitat split had only weak effects on species richness or individual species distributions. Mechanisms underlying road effects on amphibians involve direct mortality, behavioral barriers to movement, and reduction in the quality of roadside habitats. Our results suggest that the negative effects of roads on amphibians occur across broad geographic regions, affecting even common species, and they underscore the importance of developing effective strategies to mitigate the impacts of roads on amphibian populations.
\end{abstract}

(c) 2014 Elsevier Ltd. All rights reserved.

\section{Introduction}

Because landscape modification is one of the main drivers of global amphibian declines (Stuart et al., 2004; Cushman, 2006; Wake and Vredenburg, 2008), effective amphibian conservation

\footnotetext{
* Corresponding author. Tel.: +1 315781 4602; fax: +1 3157813860 . E-mail address: cosentino@hws.edu (B.J. Cosentino).
}

will likely require management at large spatial scales (Semlitsch, 2000). Species distributions are generally related to two aspects of landscape structure: the types and amounts of habitat available (habitat composition), and the spatial arrangement of habitat (habitat configuration; Turner, 2005). In temperate forest biomes, amphibian occupancy is often greatest in landscapes that have (1) high forest cover (Gibbs, 1998a; Guerry and Hunter, 2002; Porej et al., 2004; Herrmann et al., 2005), (2) low cover by urban infrastructure (Knutson et al., 1999; Lehtinen et al., 1999; Rubbo 
and Kiesecker, 2005; Pillsbury and Miller, 2008; Hamer and Parris, 2011), (3) few discontinuities between breeding and non-breeding habitats (Guerry and Hunter, 2002; Becker et al., 2007, 2010), and (4) high population connectivity (Sjögren Gulve, 1994; Houlahan and Findlay, 2003; Mazerolle et al., 2005; Werner et al., 2007; Cosentino et al., 2011).

Roads may play a particularly significant role in limiting amphibian distributions. Roads directly replace wetland and upland habitat, and they can lower the quality of adjacent habitat by creating edge effects (Marsh and Beckman, 2004) and causing run-off of deicing salts (Karraker et al., 2008). Because amphibians move slowly, individuals are also susceptible to direct mortality when moving across roads (Fahrig et al., 1995; Mazerolle, 2004; Beebee, 2013). For species with biphasic life cycles, roads can increase mortality risk during breeding migrations, ultimately increasing the probability of local extinction (Gibbs and Shriver, 2005). Fragmentation by roads at landscape scales can decrease metapopulation viability by constraining dispersal among populations (Hels and Nachmann, 2002).

Although previous studies have been critical for identifying how landscape structure and road disturbance affect amphibian distributions, these studies have varied widely in spatial extent and led to conclusions that are often site- and species-specific (Cushman, 2006). We examined effects of landscape structure and road disturbance on the distribution and richness of pondbreeding frogs and toads across the central and eastern U.S. through a multi-institutional, undergraduate research project. Approximately 200 undergraduate students in biology and environmental science courses from a network of universities compiled data from the North American Amphibian Monitoring Program (NAAMP), a database of amphibian occupancy collected by citizen scientists. NAAMP uses a standard methodology to collect occupancy data (Weir and Mossman, 2005), so we were able to investigate whether effects of landscape structure and roads on amphibian distributions and species richness are consistent across species and regions.

We addressed three questions chosen at the outset of the project: (1) What aspects of habitat composition and road disturbance best explain anuran occupancy and species richness? (2) Are the effects of road disturbance on anurans more associated with road density or traffic volume? (3) Does the separation of wetlands from upland forest by intervening land use (i.e. habitat split; Becker et al., 2007) threaten amphibian persistence? Because auditory chorus surveys were used to determine anuran presence, relationships between traffic volume and anuran distributions or species richness could be due to detection bias associated with noise during surveys. We used multiple metrics of species richness, estimation of detection probabilities, and structural equation models (SEM; Grace et al., 2010) to determine whether effects of traffic volume were due to interfering noise during surveys. We also used SEMs to disentangle correlations among landscape features characteristic of urban areas.

\section{Materials and methods}

\subsection{Sampling sites and NAAMP data}

NAAMP is a citizen-science monitoring initiative organized by the U.S. Geological Survey (Weir and Mossman, 2005). Trained observers are assigned randomly-selected driving routes within their state (Weir and Mossman, 2005). Observers initially traverse routes during the daytime and select 10 sampling locations (hereafter "stops") at least $0.5 \mathrm{~km}$ apart where bodies of water are visible within $200 \mathrm{~m}$ of the road. Observers conduct 3-4 surveys at each stop per year during pre-determined time windows that span the breeding season of most anurans in the region. At each stop, observers get out of their cars and record any anuran species heard over a 5-min survey period. During each survey, observers also record the number of cars passing by on the road and whether or not noise was present that might interfere with counting or identifying anuran calls.

Undergraduate students from nine biology and environmental science courses compiled anuran data from 1617 NAAMP stops along 406 routes in 13 states (Table A1; latitudinal extent 27.2691 to 48.5939 ; longitudinal extent -98.1681 to -70.8769 ). Students characterized landscape structure within 1-km buffers around each stop. To avoid spatial overlap in landscape indices among stops, we compiled data only for stops 1, 4, 7, and 10 within each route, ensuring stops were $\geqslant 2 \mathrm{~km}$ apart. For these stops, we first condensed the raw anuran data into summary measures for all surveys of a stop. For each stop, we calculated total number of surveys, proportion of surveys in which interfering noise was recorded, presence or absence of each species across all surveys, total number of species present (species richness) across all surveys, and mean number of cars passing by during anuran counts (traffic volume). Surveys occurred from 1994 to 2012, and the median number of surveys per stop was 12 (range $=1-45$ ).

We modified the raw NAAMP data in two ways to increase their quality. First, we interfaced county-level distribution maps from the National Amphibian Atlas (USGS, 2012) with the NAAMP survey locations and excluded any data from outside a species' known range. Thus, occupancy for each species was considered only within counties where the species was previously known to exist. There were 40 species observed across all stops after excluding data from outside a species' known, native range (Table A2). Second, we combined occupancy data for gray treefrog species (Hyla chrysoscelis and Hyla versicolor) because their calls can be difficult to distinguish.

\subsection{Landscape variables}

Landscape variables for each NAAMP stop were characterized by students using qGIS or ArcGIS software to import spatial layers from the National Land Cover Database (NLCD; Fry et al., 2011), the National Wetlands Inventory (NWI), and Open Street Map (OSM). Using these layers, students calculated the following within a buffer with a 1-km radius around each NAAMP stop: proportion of land forested, agricultural, and developed, total wetland area, total linear road length (hereafter "road density"), and total number of habitat types represented (according to NLCD classifications). Habitat split was characterized visually by whether wetlands within $200 \mathrm{~m}$ of the stop were separated by an intervening land type (i.e. road, agricultural field, or developed land) from upland forest. State-specific data were divided among participating classes (Table A1).

Several layers of quality control were applied to the landscape data to maximize their accuracy. First, two students independently compiled data for each stop, and these students were required to reach consensus before data were entered into the database. Second, entered values for each variable were sorted by magnitude and unusually high or low values were checked for accuracy by a different set of students or by an instructor. Third, a randomly chosen selection of stops was checked for accuracy for each landscape variable. Where independent calculation differed from the entered value more than $10 \%$ of the time, variables were excluded from the analysis. The only variable to fail this criterion was the number of different habitat types within the $1-\mathrm{km}$ buffer (error rate $=23 \%$ ); error rates for the other variables were $\leqslant 7 \%$. The number of different habitats was quantified by having students record the number of habitats observed visually within the $1-\mathrm{km}$ buffer around stops, 
and color codes representing habitat types were sometimes difficult to differentiate.

\subsection{Species richness}

Raw species richness was highly variable at large geographic scales, with some counties in northern Minnesota having only four species and counties in the South Carolina coastal plain having up to 31 species. Following previous studies that observed high correlations between amphibian species richness and net primary productivity (NPP) at broad geographic scales (e.g. Buckley and Jetz, 2007; Pyron and Wiens, 2013), we first performed Poisson regressions of raw species richness on NPP data obtained from FAO GeoNetwork (http://www.fao.org/geonetwork). We excluded data from all stops with $<9$ total surveys to minimize false negatives and then included total number of surveys in Poisson regressions with NPP to account for survey effort. Residuals from Poisson regressions with number of surveys and NPP were then used in subsequent analyses of the effects of landscape features on anuran species richness. Exploratory analyses using raw species richness in place of residuals yielded similar results for model comparisons, albeit with poorer model fits. Similarly, models that included NPP as a covariate (instead of using residuals from the Poisson regression) and total surveys as a covariate or offset variable produced nearly identical rankings of models.

To ensure that results for species richness were robust to inclusion criteria and detectability issues, we analyzed three additional metrics of species richness. First, we analyzed richness including only surveys in which noise was noted as absent. For this analysis, we did not exclude stops with $<9$ surveys without noise because occasional non-noisy surveys of stops that were usually noisy could provide useful information about anuran occupancy at these stops. Second, we analyzed species richness only for a subset of nine species with cumulative detection probability $\geqslant 0.9$ (see below). For this analysis we included data from all stops with at least 18 surveys, the minimum number needed to ensure cumulative detection probability of the least detectable species was $\geqslant 0.9$. Third, we determined whether any species found at $<20 \%$ of all possible stops within its known range was present at each stop ("rare species presence"). For this metric, we excluded all stops with $<9$ surveys. For the datasets with non-noisy surveys and species with high detection probability, the response variable was residuals from a regression of species richness on NPP and the total number of surveys. For rare species presence, the response variable was whether or not any rare species was present at a stop, and all models included NPP and total number of surveys as covariates.

\subsection{Species occupancy}

For analysis of individual species distributions, we focused on a subset of species with broad distributions and high detection probability. We included species that occurred in $\geqslant 3$ states and were found at $20-80 \%$ of stops within their known range. Ten species met the distribution criterion: American toad (Anaxyrus americanus), gray treefrog (H. chrysoscelis and $H$. versicolor), green treefrog (Hyla cinerea), barking treefrog (Hyla gratiosa), squirrel treefrog (Hyla squirella), American bullfrog (Lithobates catesbeianus), green frog (Lithobates clamitans), pickerel frog (Lithobates palustris), southern leopard frog (Lithobates sphenocephalus), and wood frog (Lithobates sylvaticus). Spring peeper (Pseudacris crucifer) was excluded because it was found at $83 \%$ of the stops and therefore unlikely to show obvious patterns related to land use. A variety of rare or spatially restricted species were excluded because their distributions were too narrow.

We included species that had cumulative detection probability (i.e. probability of detecting a species at a stop at least once across all surveys and years, given the species occurred at the stop) $\geqslant 0.90$. To estimate cumulative detection probability for each species, we first used occupancy modeling and program PRESENCE to estimate survey-specific detection probabilities at each stop (MacKenzie et al., 2006; see Text A1 and Tables A3-A6). We then estimated the number of surveys $(n)$ required for a cumulative detection probability $\left(p_{c}\right)$ of 0.9 by setting $p_{c}=0.9$ and solving for $n$ in the equation

$n=\frac{\log \left(1-p_{c}\right)}{\log (1-\bar{p})}$

where $\bar{p}$ is the average detection probability across all stops and surveys. For the ten species that met the distribution criterion, the required number of surveys to achieve $p_{c} \geqslant 0.9$ ranged from six (H. chrysoscelis/versicolor, $H$. cinerea, and L. clamitans) to 24 ( $H$. squirella; Table A6). We excluded stops that had fewer than the minimum number of surveys to achieve $p_{c} \geqslant 0.9$ for each species, and we used the occupancy data from the remaining stops for our analyses. We excluded squirrel treefrog from occupancy analysis because detection rate was low and few stops had sufficient surveys to achieve $p_{c} \geqslant 0.9$. This left nine species for individual analysis.

\subsection{Data analysis}

We employed generalized linear mixed models (GLMMs) to evaluate how habitat composition, road disturbance, and habitat split affected species richness and occupancy of species with $p_{c} \geqslant 0.9$. Response variables included total richness, richness from non-noisy surveys, richness for and occupancy of highly detectable species $\left(p_{c} \geqslant 0.9\right)$, and rare species presence. We specified a Gaussian error distribution and identity link for residuals of species richness metrics, and we specified a binomial error distribution and logit link for rare species presence and occupancy of individual species. Because NAAMP is structured with stops nested within randomly-chosen routes, we included route as a random effect in all models to account for spatial autocorrelation and inter-observer variation. We considered using models that account for imperfect detection (MacKenzie et al., 2006) directly for our analyses, but current multi-season approaches do not allow for random effects and are not conducive to analysis of species richness.

To determine which aspects of habitat composition and road disturbance best explained species richness and occupancy of individual species (Question 1), we compared the relative support of 12 a priori models including different combinations of explanatory variables (Table 1). Variance inflation factors (VIF; Zuur et al., 2009) were used to identify multi-collinearity among explanatory variables. Proportion forest and agriculture were highly negatively correlated (VIF $=4.8$ ), so we included only proportion forest in models. Traffic and noise level were also highly positively correlated (VIF $=6.3$ ), and we used SEMs to distinguish traffic effects from noise effects (see below).

To determine whether negative effects of roads were more closely associated with traffic volume or road density (Question 2), we compared the relative support of models that included (1) only traffic volume, (2) only road density, and (3) traffic volume and road density. These models were all part of the model set used to address Question 1 (Table 1). To determine whether habitat split affected species richness and occupancy of individual species (Question 3), we compared models that included only habitat composition variables (wetland area, forest, development, road density), composition variables plus habitat split, and a null model. Habitat split was diagnosable for only a subset of stops used for analyses of habitat composition and road disturbance, so we used separate model sets to examine habitat split. 
Table 1

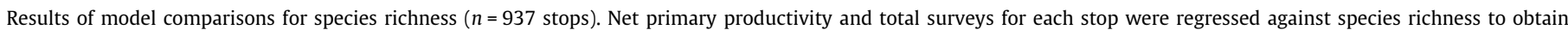

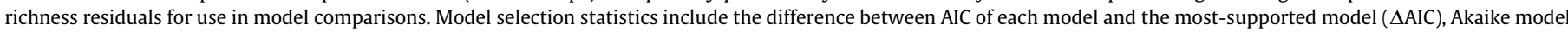
weight $(\omega)$, log-likelihood (LL), and number of parameters $(K)$. See Table A7 for model-averaged parameter estimates.

\begin{tabular}{|c|c|c|c|c|c|}
\hline Model & Terms & $\Delta \mathrm{AIC}$ & $\omega$ & LL & K \\
\hline Full & Road, traffic, development, forest, wetland & 0.00 & 0.556 & -1174.8 & 8 \\
\hline Total development & Road, traffic, development, noise & 1.17 & 0.309 & -1176.4 & 7 \\
\hline Roads & Road, traffic & 3.42 & 0.100 & -1179.5 & 5 \\
\hline Land cover & Road, development, forest, wetland & 7.11 & 0.016 & -1179.4 & 7 \\
\hline Noise & Noise & 7.28 & 0.015 & -1182.5 & 4 \\
\hline Road density & Road & 9.87 & 0.004 & -1183.8 & 4 \\
\hline Traffic & Traffic & 14.75 & 0.000 & -1186.2 & 4 \\
\hline Development & Development & 18.68 & 0.000 & -1188.2 & 4 \\
\hline Wetland area & Wetland & 19.54 & 0.000 & -1188.6 & 4 \\
\hline Natural land cover & Forest, wetland & 21.10 & 0.000 & -1188.4 & 5 \\
\hline Null & Intercept only & 26.58 & 0.000 & -1193.1 & 3 \\
\hline Forest & Forest & 26.85 & 0.000 & -1192.2 & 4 \\
\hline
\end{tabular}

Akaike's Information Criterion (AIC) was used to evaluate model support. Models were considered to have competitive support when the difference in AIC between a model and the most-supported model ( $\triangle$ AIC) was $\leqslant 2$ (Burnham and Anderson, 2002). We removed all missing observations from datasets for each model set to ensure AICs were comparable among models. Missing observations were either due to data not recorded during surveys (e.g. traffic, noise) or landscape variables excluded during quality control checks. All GLMMs were fit in R (R Core Team 2013) in the package lme4 (Bates et al., 2013). Model deviance suggested little under or over-dispersion for all models. We used the package AICcmodavg to obtain model-averaged predictions, beta coefficients, unconditional standard errors, and confidence intervals (Mazerolle, 2013).

We used SEMs to examine (1) direct and indirect effects of explanatory variables characteristic of urban areas on species richness, and (2) whether the relationship between traffic volume and species richness was potentially due to detection bias associated with noise during surveys. Species richness in the SEM was residuals from the regression of species richness on NPP and total surveys. The SEM included direct effects of traffic, noise, road density, and development on species richness. It also included effects of development on noise and traffic, an effect of traffic on noise, and covariance terms between road density and development and between road density and traffic. Because road density could influence species richness directly but should not affect noise level, this model allowed us to distinguish the indirect paths from the explanatory variables to species richness (e.g. traffic to noise to richness) from the direct effects (e.g. traffic to richness).

To fit the SEM, we first re-scaled the variables so that they would be of similar magnitude. We then fit a model that used a cluster adjustment technique for standard errors (Williams, 2000) to account for the clustering of NAAMP stops within routes. As a subsequent analysis to confirm a direct effect of traffic on species richness, we fit a second SEM that included no direct effect of traffic on richness; only an indirect effect mediated by noise. We compared AIC between models to evaluate the direct effect of traffic on species richness while accounting for correlations among the other explanatory variables. SEMs were fit with the R package lava (Holst and Budtz-Joergensen, 2012).

\section{Results}

Of the 1617 stops, 937 had enough chorus surveys and definable landscape features for analysis of species richness. Comparison among models of species richness showed that a full model containing road density, traffic volume, development, forest cover, and wetland area had the most support (Table 1). Species richness was negatively related to road density, traffic, and forest cover and positively related to wetland area and development (Fig. 1, Table A7). The positive effect of development was evident only after controlling for other variables because species richness was negatively associated with development in a model with development alone (beta $=-0.91, \mathrm{SE}=0.29$ ). A model with road density, traffic, development, and proportion of surveys with noise had competitive support. Species richness was negatively related to noise (Fig. 1, Table A7). Based on model rankings and the relative support of models with single variables, road density, traffic, and noise during surveys were the most important predictors of species richness (Table 1). When we quantified species richness using only surveys recorded as having no interfering noise, road density and traffic were the most important predictors (Tables A8 and A9). However, noise was most important for predicting the number of highly detectable species and the presence of rare species (Tables A8 and A9).

For six of nine species, the most-supported model of occupancy included road density, traffic, development, noise, or a combination of these variables (Tables 2 and A10). Occupancy was generally negatively related to road density, traffic, and noise, but the effect of development varied among species (Table A11). Occupancy for two species was most related to wetland area and forest or wetland area alone, and the null model was most supported for one species (Table 2). Wetland area generally had a positive effect on occupancy, but the effect of forest varied among species (Table A11).

For species richness, the model including both road density and traffic was more supported than models with road density or traffic alone (Table 1). This result was unchanged when we used data only from surveys without noise or when examining presence of rare species (Table A8). The traffic-only model had more support than the other models when considering only the richness of highly detectable species, but the model with road density and traffic was competitive compared to the traffic-only model $(\triangle \mathrm{AIC}=0.62$; Table $\mathrm{A} 8)$. For species occupancy, the model including road density and traffic was either more supported than singlevariable models or had competitive support for all species (Table A10).

For species richness, adding habitat split resulted in a marginal improvement in model fit over the habitat composition model (Table A12). Species richness was lower at stops that were split from upland forest (mean residual $=-0.10, \mathrm{SE}=0.09$ ) than stops that were not split (mean residual $=0.17, \mathrm{SE}=0.04$; beta $=-0.15$, $\mathrm{SE}=0.08$ ). For individual species distributions, adding habitat split improved the fit of the composition model for $L$. sylvaticus and marginally so for $H$. cinerea, but not for other species (Table A13). Occupancy was lowest at stops that were split for $L$. sylvaticus (beta $=-1.53, \mathrm{SE}=0.53$ ) and $H$. cinerea (beta $=-1.73, \mathrm{SE}=0.93$ ). 

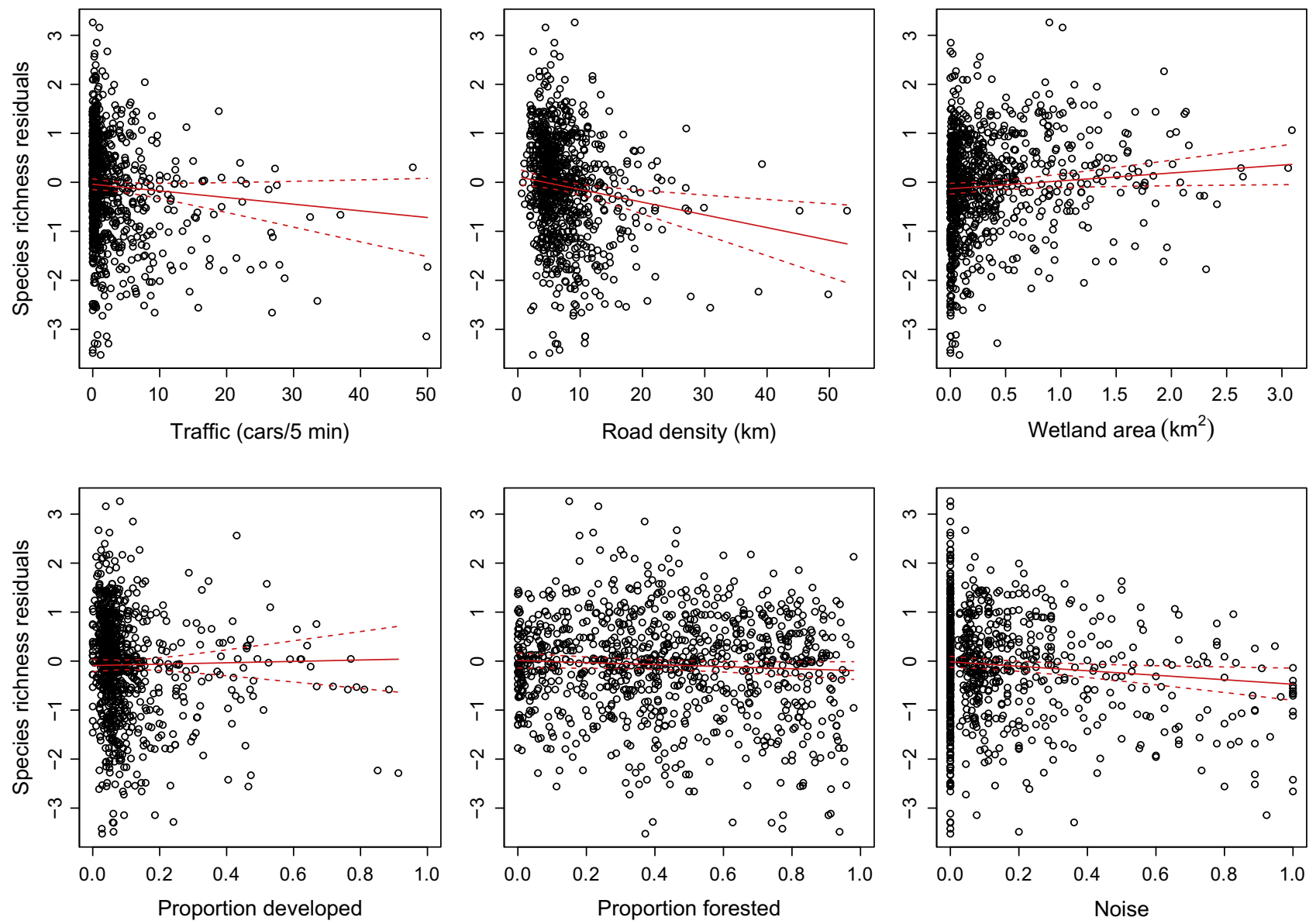

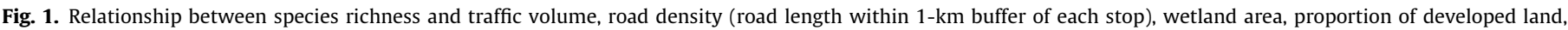

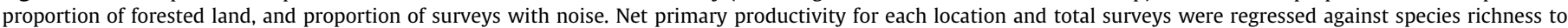

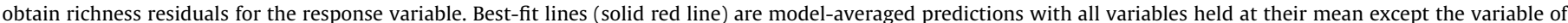

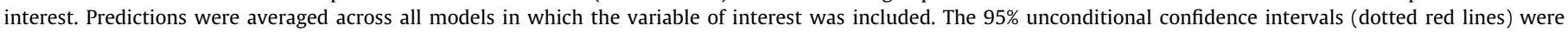

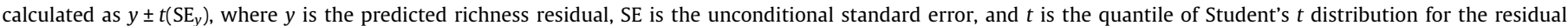
degrees of freedom. (For interpretation of the references to color in this figure legend, the reader is referred to the web version of this article.)

Table 2

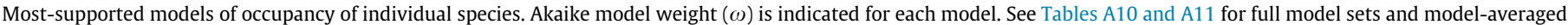
parameter estimates.

\begin{tabular}{|c|c|c|c|}
\hline Species & Model & Terms & $\omega$ \\
\hline Anaxyrus americanus & Total development & Road, traffic, development, noise & 0.579 \\
\hline Hyla chrysoscelis/versicolor & Road density & Road & 0.382 \\
\hline Hyla cinerea & Natural land cover & Forest, wetland & 0.571 \\
\hline Hyla gratiosa & Null & Intercept only & 0.171 \\
\hline Lithobates catesbeianus & Roads & Road, traffic & 0.428 \\
\hline Lithobates clamitans & Noise & Noise & 0.857 \\
\hline Lithobates palustris & Noise & Noise & 0.587 \\
\hline Lithobates sphenocephalus & Noise & Noise & 0.201 \\
\hline Lithobates sylvaticus & Wetland area & Wetland & 0.528 \\
\hline
\end{tabular}

Model fit for the SEM was good according to the root mean square error of approximation $(P=0.28)$, although there was slight lack-of-fit based on the model chi-square $\left(\chi^{2}=4.48, \mathrm{df}=1\right.$, $P=0.034)$. The model indicated that anuran species richness depended on road density, traffic, and development (Fig. $2 ; R^{2}$ for species richness residuals $=8.7 \%$ ). There were direct negative effects of both road density and traffic on species richness, but there was no direct effect of noise (Fig. 2). Development had a small, positive effect on species richness after accounting for negative effects of road density and traffic (Fig. 2). An alternative SEM with no direct effect of traffic (only an indirect effect mediated by noise) had a poorer fit to the data $(\triangle \mathrm{AIC}=13.03)$, again suggesting a direct effect of traffic on species richness.

\section{Discussion}

Our results showed that anuran species richness and occupancy were related to road disturbance, wetland area, and upland habitat composition across the eastern and central U.S. Associations between habitat composition and occupancy were largely species-specific, which supports previous findings at smaller spatial 


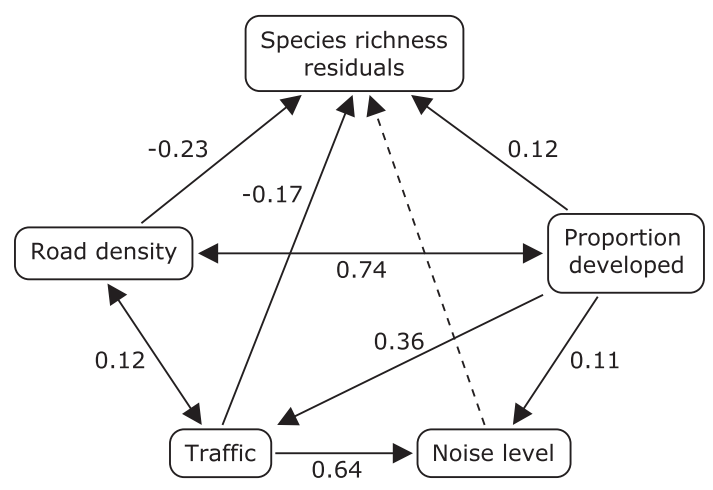

Fig. 2. Schematic of structural equation model of species richness. Net primary productivity for each location and total surveys were regressed against species richness to obtain richness residuals for the response variable. Model includes effects of road density, proportion of developed land within $1 \mathrm{~km}$ of stops, traffic volume, and proportion of surveys with noise. Single-headed arrows indicate causal relationships, and double-headed arrows indicate correlations. Coefficients are given for each pathway significant at $P<0.05$. Solid line indicates coefficients that were significant at $P<0.05$, and dashed line indicates coefficients that were not significant.

scales (Cushman, 2006). However, our analyses yielded two novel insights into general effects of landscape structure on anuran communities. First, we found that road disturbance was almost universally important in that it constrained total species richness and the distribution of most species. Second, we found that once the negative road effects were accounted for, low to moderate levels of development had a positive effect on anuran species richness.

\subsection{Road disturbance}

Species richness and the distribution of most anurans were related negatively to measures of road disturbance within $1 \mathrm{~km}$ of stops. This is consistent with previous site-specific studies showing that road disturbance interrupts processes that are important for amphibian persistence (Fahrig et al., 1995; Carr and Fahrig, 2001; Karraker et al., 2008). A model with both traffic volume and road density was more supported than single-variable models for species richness, and the SEM indicated that traffic volume and road density both had independent effects on species richness. Furthermore, the model with both road variables was competitive for all species distributions. Thus, we conclude that both aspects of road disturbance were important for explaining anuran richness and distributions.

Several mechanisms could explain why traffic volume and road density consistently limit anuran distributions across geographic locations. First, roads can increase mortality due to collisions with vehicles (Mazerolle, 2004). Every species in our analysis moves over land during breeding migrations or dispersal and - all else being equal - mortality rates generally increase as traffic volume increases (Fahrig et al., 1995; Sutherland et al., 2010). Road-associated mortality may also shift the age structure of populations resulting in smaller females with lower fecundity (Karraker and Gibbs, 2011). Second, roads may create physical or behavioral barriers to movement (Bouchard et al., 2009). These barrier effects can make seasonal migrations between wetland and upland habitats difficult (Gibbs, 1998b) or can disrupt dispersal and connectivity within a metapopulation (Hels and Nachmann, 2002). Barrier effects would be expected to scale with road density within the dispersal range of a species. Third, deicing salt that runs off into wetlands near roads has been shown to decrease survival of amphibians during embryonic and larval life stages (Sanzo and Hecnar, 2006; Karraker et al., 2008). An a posteriori analysis showed that the negative effect of traffic on species richness was somewhat stronger at northern sites with cold winters (beta $=-0.025, \mathrm{SE}=0.007$ ) than at southern sites with mild winters (beta $=-0.010, \mathrm{SE}=0.009$ ), which is consistent with the more extensive use of deicing salts in northern states.

An alternative explanation for associations between road disturbance and anuran distributions is that traffic volume and road density were confounded with habitat composition. For example, extensive road networks and high traffic volume can be characteristic of urban areas that have limited wetland and upland habitat. However, we did not find strong correlations between measures of road disturbance and the amount of wetland or forest habitat, and the SEM showed that road density and traffic had direct effects on species richness that were independent of the proportion of developed land. This indicates that associations between road disturbance and anuran distributions are not completely due to lack of habitat in urbanized landscapes.

It is possible that the negative relationship between species richness and traffic volume was caused by detection bias due to high noise during surveys. Noise was the most supported singlevariable model of species richness, and it was the most important variable for explaining occupancy for three species and presence of rare species. In addition, noise was the most important variable for predicting the richness of species that had high estimated detection probabilities. Because detection probabilities were high, it is unlikely that noise was a source of strong detection bias. Noise may have reduced species richness directly by masking breeding choruses and reducing breeding success (Bee and Swanson, 2007) or by acting as a negative cue during habitat selection. Alternatively, the relationship between noise and species richness may have been due to covariance with traffic. Indeed, the SEM confirmed that traffic increased noise during surveys, but traffic still had a direct negative effect on species richness. In contrast, there was no evidence for a direct effect of noise on species richness, and an alternative SEM without a direct effect of traffic was not supported. Furthermore, in analyses that excluded surveys with recorded noise, traffic volume and road density were the most important predictors of species richness. These results indicated that the relationship between traffic and species richness was unlikely to be solely a byproduct of detection bias.

There are important limitations to our analysis of road disturbance. First, we only examined the effects of road density and traffic. Roads vary in surface type and width, and these factors could be important for affecting mortality risk, movements, and the quality of roadside wetlands due to variation in runoff. Second, because NAAMP surveys are conducted along roads, there were no roadless sites in our study. If anurans are more likely to occupy wetlands away from roads than near roads, we could be underestimating the effects of road disturbance. Nevertheless, NAAMP survey sites are not unrepresentative of the eastern and central U.S., where typically $40-80 \%$ of all land is $<400 \mathrm{~m}$ from a road (Riitters and Wickham, 2003).

\subsection{Habitat composition and habitat split}

Our results are consistent with previous studies showing that upland habitat composition limits anuran distributions and species richness (e.g. Guerry and Hunter, 2002; Porej et al., 2004; Hamer and Parris, 2011). Based on model rankings, species richness and occupancy for most species were more closely related to upland habitat composition (forest or developed land) than to wetland area. This strongly supports the idea that conserving upland terrestrial habitat is essential for semi-aquatic amphibians to complete their life cycles (Semlitsch and Bodie, 2003; Rittenhouse and Semlitsch, 2007).

Although upland habitat composition was broadly important, specific associations between occupancy and upland habitat were 
not as consistent as the negative effects of road disturbance. Species distributions were positively or negatively related to forest, the latter likely reflecting preference for open habitats. Furthermore, both the GLMMs and the SEM indicated that species richness had a small but positive relationship with development after we accounted for the negative effects of traffic and road density. It is possible that low to moderate levels of development may add breeding sites to the landscape (e.g. farm and garden pools, retention ponds), thereby increasing occupancy of common species and species richness. Our development metric included land classified as "Developed, Open Space" (e.g., parks, lawns) in addition to impervious surfaces. Furthermore, the vast majority of survey sites had $<20 \%$ development within $1 \mathrm{~km}$. Graphically, it appears that sites with high proportions of developed land (e.g. $>60 \%$ ) may have lower species richness (Fig. 1), but the number of these sites was too few for breakpoint analysis to pick up this effect.

The negative effect of habitat split on species richness is consistent with previous findings for aquatic-breeding species in tropical ecosystems (Becker et al., 2007), and it supports the notion that wetland-upland linkages can be critical for facilitating breeding migrations. However, the effect of habitat split on species richness was not particularly strong, and habitat split constrained the distribution of only two of nine species. The weak association between habitat split and species richness may stem from defining forest as upland habitat, which may not be applicable to species that associate with more open habitats. Furthermore, our analysis of habitat configuration was limited in that we did not consider distances between wetlands and upland habitat or connectivity to other wetlands in the landscape. Further study is needed to examine the relative impacts of habitat composition and configuration on amphibian distributions (Fahrig, 2003; Quesnelle et al., 2013).

\subsection{Strength of landscape effects}

Although the relationships we observed between anuran occupancy and richness and road disturbance were largely consistent across species, these relationships tended to have weak explanatory power. That these relationships were weak is not surprising given that we were looking for general associations between anurans and land use across species and regions. Much of the variation in species richness was explained by route $\left(R^{2}=44 \%\right.$ for fixed effect of route on species richness), and landscape effects within specific regions and for anurans with specific habitat preferences would likely be stronger. In addition, several other factors likely added noise to the observed relationships. These factors include the potential for both false positives and false negatives using the NAAMP volunteer protocol (Genet and Sargent, 2003), limited resolution and accuracy of NLCD landcover maps (Wickham et al., 2013), land use change over the course of NAAMP data collection, and errors in variable calculation by participating students. Given all these sources of noise, our conclusion is not that amphibian distributions are largely explained by road disturbance, but rather that road disturbance effects were surprisingly detectable across species and regions of the U.S.

\subsection{Conclusions}

Recent evidence suggests that amphibian declines in the U.S. are widespread, with occupancy rates declining $3.7 \%$ per year (Adams et al., 2013). Effective landscape management will be critical for reversing this trend. Despite regional variation in species assemblages and habitat preferences of individual species, our results demonstrate that road disturbance has a broad influence on the spatial distribution and local diversity of anurans in the eastern and central U.S.
Successful amphibian conservation will likely require minimizing the impacts of future road projects and mitigating the impacts of current roads. Mitigation for amphibians can take the form of tunnels that allow for safe passage, fences that prevent animals from entering roads, signage to alert drivers to road-crossing zones, and reduced road salt application near wetlands (Lesbarrères and Fahrig, 2012; Beebee, 2013). Mitigation can be targeted by identifying hotspots of road mortality (Langen et al., 2009), which usually correspond to areas where roads pass close to wetlands, but are not necessarily the areas with the highest traffic (Eberhardt et al., 2013). Further research on the mechanisms underlying road effects on amphibians (e.g. roadkill mortality, deicing salts, barrier effects) could help clarify which kinds of mitigation efforts are most likely to be successful in any given situation.

\section{Acknowledgements}

We are grateful to Stephanie Hampton and the National Center for Ecological Analysis and Synthesis (NCEAS) for facilitating this project. Jim Regetz and Thomas Hetmank (NCEAS) provided essential technical support. Linda Weir (NAAMP) helped us understand NAAMP data and provided comments on an earlier draft of the manuscript, and we thank Marc Mazerolle for advice about model averaging. We thank students from classes at Anoka Ramsey Community College, Clarkson University, Eckerd College, Hobart and William Smith Colleges, University of Rhode Island, University of South Carolina Salkehatchie, Utah State University, Virginia Commonwealth University, and Warren Wilson College for contributing data. This research was funded with a grant from the NSF Transforming Undergraduate Education in Science Program (TUES) to DMM.

\section{Appendix A. Supplementary material}

Supplementary data associated with this article can be found, in the online version, at http://dx.doi.org/10.1016/j.biocon.2014.09. 027.

\section{References}

Adams, M.J. et al., 2013. Trends in amphibian occupancy in the United States. PLoS ONE 8 (5), e64347.

Bates, D., Maechler, M., Bolker, B., Walker, S. 2013. Ime4: Linear Mixed-Effects Models Using Eigen and S4. R package version 1.0-5. <http://CRAN.Rproject.org/package $=$ lme $4>$.

Becker, C.G., Fonesca, C.F., Haddad, C.F.B., Batista, R.F., Prado, P.I., 2007. Habitat split and the global decline of amphibians. Science 318, 1775-1777.

Becker, C.G., Fonesca, C.R., Haddad, C.F.B., Prado, P.I., 2010. Habitat split as a cause of local population declines of amphibians with aquatic larvae. Conserv. Biol. 24, 287-294.

Bee, M.A., Swanson, E.M., 2007. Auditory masking of anuran advertisement calls by road traffic noise. Anim. Behav. 74, 1765-1776.

Beebee, T.J.C., 2013. Effects of road mortality and mitigation measures on amphibian populations. Conserv. Biol. 4, 657-668.

Bouchard, J., Ford, A.T., Eigenbrod, F.E., Fahrig, L., 2009. Behavioral responses of northern leopard frogs (Rana pipiens) to roads and traffic: implications for population persistence. Ecol. Soc. 14, 23.

Buckley, L.B., Jetz, W., 2007. Environmental and historical constraints on global patterns of amphibian richness. Proc. Royal Soc. B 274, 1167-1173.

Burnham, K.P., Anderson, D.R., 2002. Model Selection and Multimodel Inference: A Practical Information-Theoretic Approach, second ed. Springer, New York, NY, USA.

Carr, L.W., Fahrig, L., 2001. Effect of road traffic on two amphibian species of differing vagility. Conserv. Biol. 15, 1071-1078.

Cosentino, B.J., Schooley, R.L., Phillips, C.A., 2011. Spatial connectivity moderates the effect of predatory fish on salamander metapopulation dynamics. Ecosphere 2 (8), art95.

Cushman, S.A., 2006. Effects of habitat loss and fragmentation on amphibians: a review and prospectus. Biol. Conserv. 128, 231-240.

Eberhardt, E., Mitchell, S., Fahrig, L., 2013. Road kill hotspots do not effectively indicate mitigation locations when past road kill has depressed populations. J. Wildl. Manage. 77, 1353-1359. 
Fahrig, L., 2003. Effects of habitat fragmentation on biodiversity. Annu. Rev. Ecol. Syst. $34,487-515$.

Fahrig, L., Pedlar, J.H., Pope, S.E., Taylor, P.D., Wegner, J.F., 1995. Effect of road traffic on amphibian density. Biol. Conserv. 73, 177-182.

Fry, J. et al., 2011. Completion of the 2006 national land cover database for the conterminous United States. Photogramm. Eng. Remote Sens. 77, 858-864.

Genet, K.S., Sargent, L.G., 2003. Evaluation of methods and data quality from a volunteer-based amphibian call survey. Wildl. Soc. Bull. 31, 703-714.

Gibbs, J.P., 1998a. Distribution of woodland amphibians along a forest fragmentation gradient. Landscape Ecol. 13, 263-268.

Gibbs, J.P., 1998b. Amphibian movements in response to forest edges, roads, and streambeds in southern New England. J. Wildl. Manage. 62, 584-589.

Gibbs, J.P., Shriver, W.G., 2005. Can road mortality limit populations of poolbreeding amphibians? Wetlands Ecol. Manage. 13, 281-289.

Grace, J.B., Anderson, T.M., Olff, H., Scheiner, S.M., 2010. On the specification of structural equation models for ecological systems. Ecol. Monogr. 80, 67-87.

Guerry, A.D., Hunter, M.L., 2002. Amphibian distributions in a landscape of forests and agriculture: an examination of habitat composition and configuration. Conserv. Biol. 16, 745-754.

Hamer, A.J., Parris, K.M., 2011. Local and landscape determinants of amphibian communities in urban ponds. Ecol. Appl. 21, 378-390.

Hels, T., Nachmann, G., 2002. Simulating viability of a spadefoot toad Pelobates fuscus metapopulation in a landscape fragmented by a road. Ecography 25, 730 744.

Herrmann, H.L., Babbitt, K.J., Baber, M.J., Congalton, R.G., 2005. Effects of landscape characteristics on amphibian distribution in a forest-dominated landscape. Biol. Conserv. 123, 139-149.

Holst, K.H., Budtz-Joergensen, E., 2012. Linear latent variable models. Comput. Statistics, <http://dx.doi.org/10.1007/s00180-012-0344-y>.

Houlahan, J.E., Findlay, C.S., 2003. The effects of adjacent land use on wetland amphibian species richness and community composition. Can. J. Fish. Aquat. Sci. 60, 1078-1094.

Karraker, N.E., Gibbs, J.P., 2011. Contrasting road effect signals in reproduction of long-versus short-lived amphibians. Hydrobiologia 664, 213-218.

Karraker, N.E., Gibbs, J.P., Vonesh, J.R., 2008. Impacts of road deicing salt on the demography of vernal pool-breeding amphibians. Ecol. Appl. 18, 724-734.

Knutson, M.G., Sauer, J.R., Olsen, D.A., Mossman, M.J., Hemasath, L.M., Lannoo, M.J., 1999. Effects of landscape composition and wetland fragmentation on frog and toad abundance and species richness in Iowa and Wisconsin, USA. Conserv. Biol. 13, 1437-1446.

Langen, T.A., Ogden, K.M., Schwarting, L.L., 2009. Predicting hot spots of herpetofauna road mortality along highway networks. J. Wildl. Manage. 73, 104-114.

Lehtinen, R.M., Galatowitsch, S.M., Tester, J.R., 1999. Consequences of habitat loss and fragmentation for wetland amphibian assemblages. Wetlands 19, 1-12.

Lesbarrères, D., Fahrig, L., 2012. Measures to reduce population fragmentation by roads: what has worked and how do we know? Trends Ecol. Evol. 27, 374-380.

MacKenzie, D.I., Nichols, J.D., Royle, J.A., Pollock, K.H., Bailey, L.L., Hines, J.E., 2006. Occupancy Estimation and Modeling: Inferring Patterns and Dynamics of Species Persistence. Academic Press, Burlington, Massachusetts, USA.

Marsh, D.M., Beckman, N.G., 2004. Effects of forest roads on the abundance and activity of terrestrial salamanders. Ecol. Appl. 14, 1882-1891.

Mazerolle, M.J., 2004. Amphibian road mortality in response to nightly variations in traffic intensity. Herpetologica 60, 45-53.

Mazerolle, M.J., 2013. AICcmodavg: Model Selection and Multimodel Inference Based on (Q)AIC(c). R Package Version 1.28. <http://CRAN.R-project.org/ package=AICcmodavg $>$.
Mazerolle, M.J., Desrochers, A., Rochefort, L., 2005. Landscape characteristics influence pond occupancy by frogs after accounting for detectability. Ecol. Appl. 15, 824-834.

Pillsbury, F.C., Miller, J.R., 2008. Habitat and landscape characteristics underlying anuran community structure along an urban-rural gradient. Ecol. Appl. 18, 1107-1118.

Porej, D., Micacchion, M., Hetherington, T.E., 2004. Core terrestrial habitat for conservation of local populations of salamanders and wood frogs in agricultural landscapes. Biol. Conserv. 120, 399-409.

Pyron, R.A., Wiens, J.J., 2013. Large-scale phylogenetic analyses reveal the causes of high tropical amphibian diversity. Proc. Royal Soc. B 280, 20131622.

Quesnelle, P.E., Fahrig, L., Lindsay, K.E., 2013. Effects of habitat loss, habitat configuration and matrix composition on declining wetland species. Biol. Conserv. 160, 200-208.

R Core Team, 2013. R: A Language and Environment for Statistical Computing. R Foundation for Statistical Computing, Vienna, Austria.

Riitters, K.H., Wickham, J.D., 2003. How far to the nearest road? Front. Ecol. Environ. $1,125-129$.

Rittenhouse, T.A.G., Semlitsch, R.D., 2007. Distribution of amphibians in terrestrial habitat surrounding wetlands. Wetlands 27, 153-161.

Rubbo, M.J., Kiesecker, J.M., 2005. Amphibian breeding distribution in an urbanized landscape. Conserv. Biol. 19, 504-511.

Sanzo, D., Hecnar, S.J., 2006. Effects of road de-icing salt ( $\mathrm{NaCl})$ on larval wood frogs (Rana sylvatica). Environ. Pollut. 140, 247-256.

Semlitsch, R.D., 2000. Principles for management of aquatic-breeding amphibians. J. Wildl. Manage. 64, 615-631.

Semlitsch, R.D., Bodie, J.R., 2003. Biological criteria for buffer zones around wetlands and riparian habitats for amphibians and reptiles. Conserv. Biol. 17, 1219-1228.

Sjögren Gulve, P., 1994. Distribution and extinction patterns within a northern metapopulation of the pool frog, Rana lessonae. Ecology 75, 1357-1367.

Stuart, S.A., Chanson, J.S., Cox, N.A., Young, B.E., Rodrigues, A.S.L., Fischman, D.L., Waller, R.W., 2004. Status and trends of amphibian declines and extinctions worldwide. Science 306, 1783-1786.

Sutherland, R.W., Dunning, P.R., Baker, W.M., 2010. Amphibian encounter rates on roads with different amounts of traffic and urbanization. Conserv. Biol. 24, $1626-1635$.

Turner, M.G., 2005. Landscape ecology: what is the state of the science? Annu. Rev. Ecol. Evol. Syst. 36, 319-344.

United States Geological Survey National Amphibian Atlas. 2012. Vers. 2.2, USGS Patuxent Wildlife Research Center, Laurel, Maryland. <www.pwrc.usgs.gov/ naa>.

Wake, D.B., Vredenburg, V.T., 2008. Are we in the midst of the sixth mass extinction? a view from the world of amphibians. Proc. Natl. Acad. Sci. USA 105, $11466-11473$.

Weir, L.A., Mossman, M.J., 2005. North American Amphibian Monitoring Program. In: Lannoo, M.J. (Ed.), Amphibian Declines: the Conservation Status of United States Species. University of California Press, Berkeley, California, USA, pp. 307-313.

Werner, E.E., Yurewicz, K.L., Skelly, D.K., Relyea, R.A., 2007. Turnover in an amphibian metacommunity: the role of local and regional factors. Oikos 116, $1713-1725$.

Wickham, J.D., Stehman, S.V., Gass, L., Dewitz, J., Fry, J.A., Wade, T.G., 2013. Accuracy assessment of NLCD 2006 land cover and impervious surface. Remote Sens. Environ. 130, 294-304.

Williams, R.L., 2000. A note on robust variance estimation for cluster-correlated data. Biometrics 56, 645-646.

Zuur, A.F., Ieno, E.N., Walker, N.J., Saveliev, A.A., Smith, G., 2009. Mixed Effects Models and Extensions in Ecology with R. Springer, New York, NY, USA. 